\title{
Gallic Acid Ameliorates Atopic Dermatitis-Like Skin Inflammation Through Immune Regulation in a Mouse Model
}

\section{Guohong Hu \\ Xiansheng Zhou}

Dermatology Hospital of Jiangxi Province, Nanchang, 33000I, Jiangxi, People's Republic of China
Correspondence: Guohong $\mathrm{Hu}$ Dermatology Hospital of Jiangxi Province, No. 388 Yingbin North Road, Nanchang, 33000I, Jiangxi, People's Republic of China

Tel +86-79I-85221034

Email 2009020212@st.btbu.edu.cn
Background: Gallic acid (GA) has an anti-inflammatory effect by regulating inflammatory molecules. This study aimed to investigate the effect of GA on atopic dermatitis (AD)-like skin inflammation.

Methods: 4-dinitrochlorobenzene (DNCB) was used to induce an AD-like skin inflammation model. The effect of GA on DNCB-induced inflammation was assessed by measuring the thickness and histopathological examination of the ear. Serum IgE and TNF- $\alpha$ levels were detected. The effect of GA on lymph nodes was determined by measuring the weights and mRNA/protein expression levels of TNF- $\alpha$, IL-4, IFN- $\gamma$ and IL-17. Ratio of Treg cells and Th17 cells was also analyzed.

Results: It was found that the thickness and pathology of the ear were significantly improved by GA in the DNCB-induced mice. Serum IgE and TNF- $\alpha$ levels were significantly reduced in GA-treated model mice compared to the model group. GA treatment lowered the weight of lymph node and the expression of mRNAs of TNF- $\alpha$, IL-4, IFN- $\gamma$, and IL-17 of lymph node. In the ear, inflammatory factors (IL-4, IL-5, IL-17, or IL-23) showed a significant decrease in GA-treated model mice versus model mice, while the expression levels of IL-10 and TGF- $\beta$ showed a great increase in GA-treated model mice. ROR- $\gamma$ t showed a decrease in GA-treated model group, along with an increase expression of SOCS3.

Conclusion: GA could ameliorate AD-like skin inflammation possibly through Th17 mediated immune regulation in a DNCB-induced mouse model.

Keywords: 4-dinitrochlorobenzene, DNCB, Th17, IL-10, TGF- $\beta$, IFN- $\gamma$

\section{Introduction}

Atopic dermatitis (AD) is a common term that denotes several different types of skin disorders that are mostly present as itchy, red, swollen, and cracked skin. ${ }^{1}$ Due to its unusual pathogenesis and clinical characteristics, ${ }^{2}$ the current knowledge of $\mathrm{AD}$ is not sufficient for clinical diagnosis and treatment. At present, the firstline treatments for AD are calmodulin inhibitors, immunosuppressants, and antiinflammatory drugs as corticosteroids. ${ }^{1}$ In many cases, the therapeutic aim is to control the disease progression rather than cure it. There are currently targeted therapeutic drugs such as Dupilumab, which targeting IL-4/IL-13, mostly used for moderate-to-severe $\mathrm{AD}^{3}$ Recently, oral and topical JAK/STAT inhibitors are in Phase II and III clinical trials for the treatment of $\mathrm{AD}{ }^{4}$ However, they are expensive and for some patients are not effective. Janus kinase (JAK) inhibitors 
that effectively treat a multitude of hematologic and inflammatory diseases directly block the JAK kinase signaling pathway, inhibiting the occurrence of inflammation processes, but have not yet been approved for $\mathrm{AD}$ treatment. ${ }^{5}$ While urgent, the worldwide prevalence of $\mathrm{AD}$ is increasing with about $70 \%$ of cases occurring before the age of five. ${ }^{6}$ Thus, new strategies for $\mathrm{AD}$, especially for Children's AD, are especially needed.

Effective topical and systemic therapies can reduce certain immune pathway activation and inflammation, thus preventing the development of AD. Polyphenols are typified by antioxidant and protective effects in cardiovascular and neurological disorders. ${ }^{7,8}$ One of the most important polyphenols is gallic acid (GA), which is present in grapes, mango, and green tea, ${ }^{9}$ also known as 3,4,5-trihydroxybenzoic acid. GA was shown to have positive effects on hypertension, vascular calcification, cardiac remodeling, and fibrosis. Moreover, GA has antioxidant properties and anti-inflammatory effects that eliminate and regulate free radicals and inflammatory molecules. ${ }^{10-12}$ GA treatment can suppress the levels of proinflammatory cytokines IL-1 and IL-6, chemokines CCL-2 and CCL-7, cyclooxygenase-2 (COX-2), and matrix metalloproteinase-9 (MMP-9) on rheumatoid arthritis fibroblast-like synovial cells (FLS).$^{13}$ It was also reported that GA was able to regulate the immune response in the allergic rhinitis model of mice, thus improving allergic rhinitis. ${ }^{12}$ So, whether GA has a protective effect on the $\mathrm{AD}$ model in mice, and whether it regulates the immune response, there are no answers to these questions up to date.

In this study, we sought to investigate the effect of GA on AD. The characteristics of AD histology are dermal and epidermal inflammatory infiltrates, including eosinophils. In our study, we used 4-dinitrochlorobenzene (DNCB) application, which can effectively induce skin inflammation that mimics AD histology and symptoms, a defective skin barrier with increased penetration of allergens and pathogens. DNCB is hereby used to induce the AD-like model. ${ }^{14}$ Using this model to induce AD-like skin inflammation, GA was given to model mice to detect its effects and explore possible underlying mechanisms.

\section{Materials and Methods}

\section{Mouse Model and Treatment}

AD-like mouse model was settled according to the previous protocol, ${ }^{15}$ as illustrated in Figure 1A. Female BALB/c mice of 7 weeks were used. DNCB (Sigma,
St. Louis, MO) was dissolved with acetone/olive oil. At day $0,3,6$ mice were given $1 \%$ DNCB treatment (10 $\mu \mathrm{L}$ ) once each day, and the treatment was smeared on the right ear. Starting from day 7, 0.5\% DNCB was applied every 3 days. Control mice were treated with just acetone/ olive oil. Dexamethasone (DEX) was used as the standard positive control at the dose of $10 \mu \mathrm{g} / \mathrm{ear}^{11}{ }^{11}$ Dexamethasone application started on the 7th day, once every 3 days, 30 minutes before the challenge. GA treatment started on the 7th day once a day. On the 43rd day, the induced dermatitis was assessed, and the mice were executed for biochemical testing. GA (purity $\geq 98 \%$, Sigma Chemical Co., St. Louis, MO, USA) was dissolved in phosphate-buffered saline (PBS). The method of administration is oral gavage. The dosing concentration refers to a previous study. ${ }^{12} 20$, $40,80 \mathrm{mg} / \mathrm{kg}$ dissolved in $200 \mu \mathrm{L}$ PBS, store at $4^{\circ} \mathrm{C}$. Routine standard histology protocol was followed for tissue collection, hematoxylin and eosin (HE) staining, and histological examination.

This study was performed in strict accordance with the NIH guidelines for the care and use of laboratory animals (8th edition, NIH), and approved by Dermatology Hospital of Jiangxi Province.

\section{Evaluation of Skin Dermatitis Severity}

Scoring for dermatitis followed the method described previously. ${ }^{16}$ Total dermatitis score is the sum of 4 individual scores: oedema, erythema/haemorrhage, scaling/dryness and excoriation/erosion. For each index, the score was judged as follows: 0 , no symptoms; 1 , mild; 2, moderate; and 3, severe. All the evaluation was performed blindly.

The measurement of the thickness was done by selecting 6 microscopic fields from each mouse. The average value of 6-8 points is used as the value of a field, and the value of 6 fields is then averaged as the value of a mouse. Figures shown are from 6 mice.

\section{Weight Measurement of the Lymph Node and Spleen}

The lymph node samples were collected from mesenteric lymph nodes. The weights of the lymph nodes were measured using an electronic balance (Mettler Toledo, Columbus, Ohio, USA). The sizes and weights of the spleen and lymph nodes were compared among the groups according to the following calculations: 

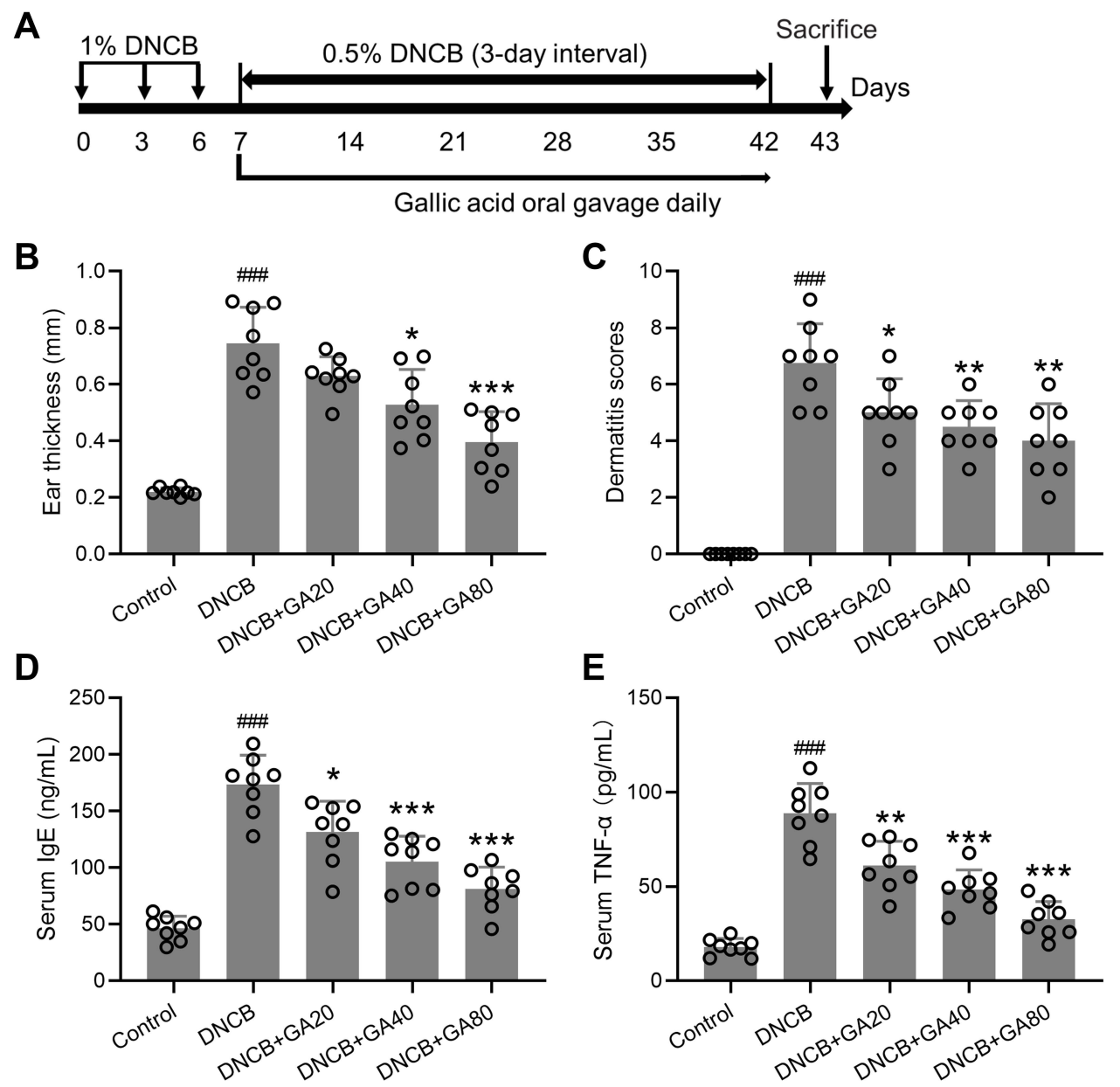

Figure I Experimental design and observation indicators. (A) Schematic diagram of the study protocol. (B) Comparisons of ear thickness at the end of experimental procedure. (C) Effects of gallic acid on atopic dermatitis-like skin severity induced by DNCB. (D and E) Effects of gallic acid on serum levels of lgE (D) and TNF- $\alpha$ (E) in atopic dermatitis-like mice. Mean \pm SD was used to shown the data. \#\# $<0.001$ compared to control, *p $<0.05$, **p $<0.0$ I, ***p $<0.00$ I compared to DNCB group. One-way ANOVA followed Dunn's multiple comparisons test.

Weight index of the spleen

$=$ weight of the spleen/body weight $\times 100 \%$

Weight index of the lymph node

$=$ weight of the lymph node/body weight $\times 100 \%$

\section{RT-qPCR and Western Blot}

RNA was isolated from lymph node tissue and skin tissue of the ear by using RNeasy Kit (Cat. 74004, Qiagen, Valencia, CA) following the manufacturer's instructions. RT-qPCR was done by using QIAGEN OneStep RT-PCR Kit following the manufacturer's instruction. Primers used were as shown in Table S1. Western blot was done followed the standard protocol using total protein extraction kit (\#2140, Chemicon, Billerica, MA) and Pierce ECL PLUS detection system (\#32132, Thermo
Fisher Scientific, Waltham, MA), antibodies used were rabbit anti $\beta$-actin (1:1000, \#4967, Cell Signaling Technology, Danvers, MA), Rat anti ROR- $\gamma \mathrm{t}(5 \mu \mathrm{g} / \mathrm{mL}$, \#14-6981-82, Thermo Fisher Scientific), Rabbit anti SOCS3 (1:1000, \#2923, Cell Signaling Technology).

To analyze the qPCR data, the $2^{-\Delta \Delta \mathrm{CT}}$ method was used. First, we verified the amplification efficiency of the target gene and the reference gene. The amplification efficiency of the target gene and the reference gene is close to $100 \%$, and the efficiency deviation between each other is within $5 \%$. For all test samples and calibration samples, the CT value of the internal reference gene is used to normalize the $\mathrm{CT}$ value of the target gene. Secondly, the $\triangle \mathrm{CT}$ value of the calibration sample is used to normalize the $\Delta \mathrm{CT}$ value of the test sample. Finally, the expression level ratio is calculated. 


\section{ELISA}

ELISA was done with the standard protocol from the manufacturer, using the following ELISA kit from Abcam (Cambridge, MA): IL-4 (ab100710), IL-5 (ab100711), IL-17 (ab204522), and IL-23 (ab119545).

\section{Flow Cytometry Analysis}

Lymphocytes were isolated from spleens with a lymphocyte separation kit (Solarbio, Beijing, China) following the manufacturer's instruction. Lymphocytes were washed with PBS and resuspended on PBS, and then the antibody of CD4-APC (553051, BD Biosciences, Franklin Lakes, NJ) was added and mixed well. After incubation at $37^{\circ} \mathrm{C}$ for 20 minutes, cells were fixed and permeabilized. The cells were further stained with antibody of FOXP3-PE (566881, BD Biosciences) or IL-17A-PE (12-7177-81, Thermo Fisher). After washing and resuspending in PBS with $10 \%$ FBS, the stained cells were analyzed by flow cytometer (BD Accuri C6 Flow Cytometer, BD Biosciences). The number of Treg cells (CD4+/FOXP3+) or Th17 cells (CD4+/IL-17A+) was then quantified.

\section{Statistical Analysis}

All experiments were conducted in triplicate and repeated at least 3 times. Statistical analyses were performed using the GraphPad Prism 5 software (version 5.03; GraphPad software, Inc., San Diego, CA, USA). P value was calculated by one-way ANOVA analysis of variance followed by Dunn's multiple comparisons test, or Kruskal-Wallis test following by Mann-Whitney $U$-tests. Significance was set at $\mathrm{P}<0.05$ for all comparisons.

\section{Results}

\section{Effect of GA on DNCB-Induced AD-Like Mouse Model}

DNCB is commonly used to induce AD-like dermatitis in mice, and we used DNCB administration for 5 weeks to build an AD model (Figure 1A). After 3 times of DNCB treatment, GA was applied for 5 consecutive weeks at 3 doses $(20,40,80 \mathrm{mg} / \mathrm{kg})$, and various indicators were tested at the end of the experiment. The effect of GA on mice with DNCB induced atopic dermatitis-like inflammation was assessed. It was found that the thickness of the ear was significantly thickened in the DNCB-treated model group, which was significantly improved by GA, and was more effective as the dose increased, with the highest dose effect being best (Figure 1B). Dexamethasone group also showed an improvement in thickness (Figure $\underline{\mathrm{S} 1 \mathrm{~A}}$ ). The same result (Figure 1C, Figure S1B) can be seen by scoring.

The elevation of serum IgE is an important feature in patients with $\mathrm{AD}$, along with IL-4, IL-13. ${ }^{17}$ The detection of serum IgE and TNF- $\alpha$ levels showed that they were significantly reduced in GA-treated groups compared to the model group, with the highest dose group approaching the control group level (Figure 1D and E). Dexamethasone treated mice also showed a decrease of these two factors (Figure S1C and D). Histopathological examination further confirmed the function of GA. In the subsequent tests, due to the remarkable effect of $80 \mathrm{mg} / \mathrm{kg}$ group, we chose this dose only for the following experiments. GAtreated DNCB-induced mice had a more normal ear skin HE staining structure (Figure 2A), and statistics showed that the epidermis thickness (Figure 2B) and dermal thickness (Figure 2C) were significantly thinner than the model group. It can be seen that GA significantly improved the symptoms of dermatitis and reduced the level of inflammatory factors in the serum.

\section{Effect of GA on Lymph Node}

Effects of GA on the weights of lymph nodes and the expression of inflammatory factors in the lymph nodes were also determined. The lymph nodes were excised on day 43 , and the weights were compared to calculate the lymph node index. Lymph node weight of DNCBtreated model group was the heaviest, while GA treatment lowered its weight (Figure 3A), the index showed the same trend (Figure 3B). For spleen, it showed the same as for lymph node (Figure S2). The total RNA was isolated from the lymph node and qRT-PCR was used to measure the RNA expression levels of TNF- $\alpha$ (Figure 3C), IL-4 (Figure 3D), IFN- $\gamma$ (Figure 3E), and IL-17 (Figure 3F). All these inflammatory factors showed a significant increase in DNCB-treated model mice, while GAtreatment decreased their expression. These data suggested that GA alleviated the inflammation induced by DNCB in mice.

\section{Effect of GA on Inflammatory Factors}

It was implicated that GA could function as an inflammatory mediator, thus affecting the expression or secretion of certain inflammatory cytokines. At the same time, mRNA from the ear was also isolated and checked for those inflammatory factors, to assess the effects of GA on the 


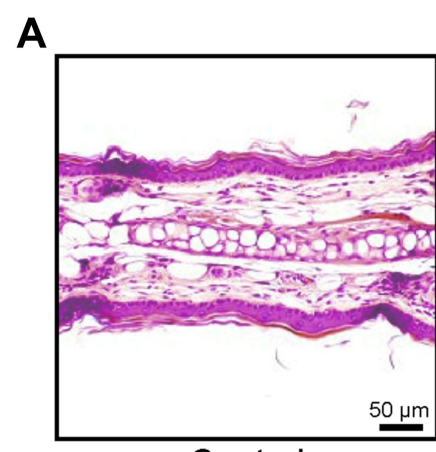

Control

B

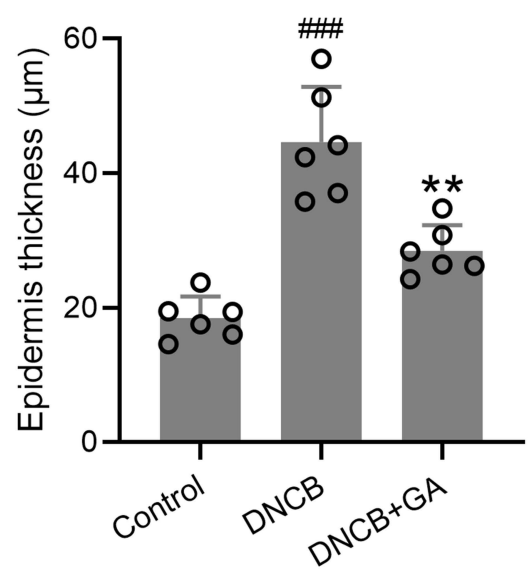

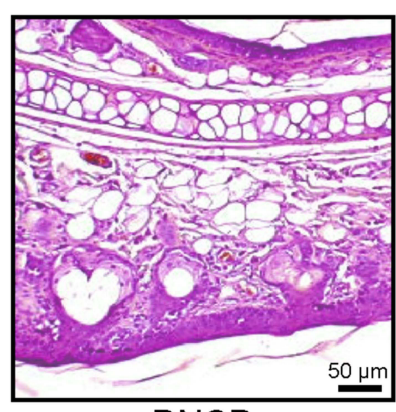

DNCB

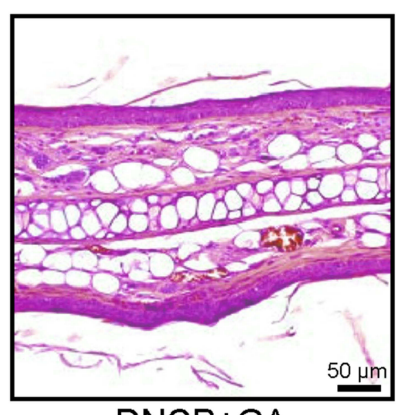

$\mathrm{DNCB}+\mathrm{GA}$

C

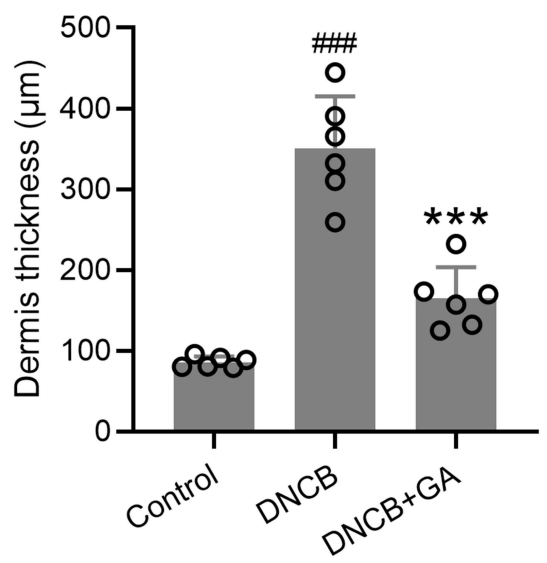

Figure 2 Histopathological observation of ear tissues from DNCB induced atopic dermatitis-like mice. (A) Representative images of hematoxylin-eosin staining for ear skin. The comparison of thickness of the epidermis and dermis (B and $\mathbf{C}$ ). Mean \pm SD was used to shown the data. \#\#p $<0.00$ I compared to control, $* * p<0.0$ I, $* * * p<0.00$ I compared to DNCB group. One-way ANOVA followed Dunn's multiple comparisons test.

expression of inflammatory factors in the ear. The ears were excised on day 43. mRNA expression levels of IL4 (Figure 4A), IL-5 (Figure 4B), IL-17 (Figure 4C) and IL23 (Figure 4D) showed an increase in DNCB-induced model group, a decrease in GA treated model group. ELISA results for these factors proved that their protein levels elevated at the same time (Figure S3). While the expression levels of IL-10 (Figure 4E) and TGF- $\beta$ (Figure 4F) showed the opposite, no significant change for IL-10, slightly decrease for TGF- $\beta$ in DNCB-induced mice. However, these two factors showed a great increase in GA-treated group. These results indicated a role of inflammatory modulation of GA in DNCB-induced ADlike inflammation.

\section{Effects of GA on the ThI7 Mediated Immune Responses}

Next, we sought to investigate if Th17 cells participated in GA mediated response. In spleens of DNCB-induced ADlike mice (Figure S4), the ratio of Th17 cells decreased dramatically, while GA treatment rescued this effect. However, the ratio of Treg cells showed the opposite change. Being as a direct activator of Th17 cell signature genes and a direct repressor of signature genes from other T cell lineages, ROR- $\gamma \mathrm{t}$ indicated the activation of Th17, ${ }^{18}$ while SOCS3 function in the differentiation of Th17 as a negative regulator. ${ }^{19}$ qRT-PCR and Western blotting were used to measure the mRNA and protein expressions of ROR- $\gamma \mathrm{t}$ and SOCS3. The expression of ROR- $\gamma \mathrm{t}$ increased in DNCB-induced model mice with an alleviation in GA-treated group (Figure $5 \mathrm{~A}-\mathrm{C}$ ). The expression of SOCS3 showed an opposite change, it decreased in DNCB-induced model group but increased with GAtreatment (Figure 5D-F). These data suggested the activation of Th17 in DNCB-induced AD-like inflammation, while GA treatment could reverse the effect.

\section{Discussion}

Since the mechanism is unknown, AD can easily be confused with eczema, which adds to the difficulty of making decisions for clinical treatment. Research on AD pathogenesis to 
A

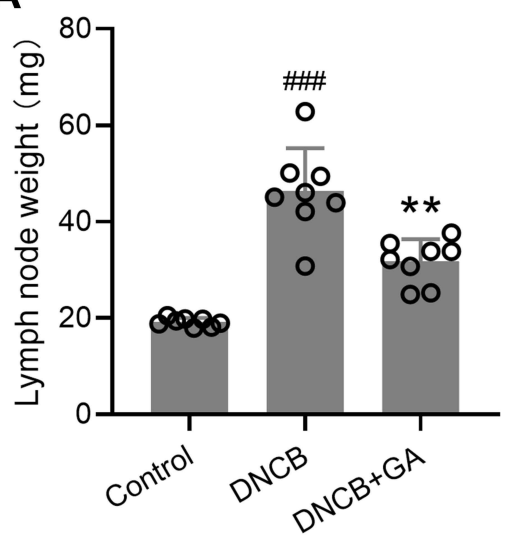

D

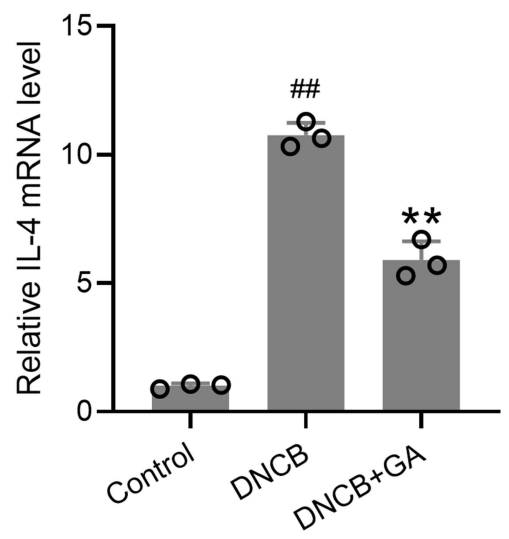

B

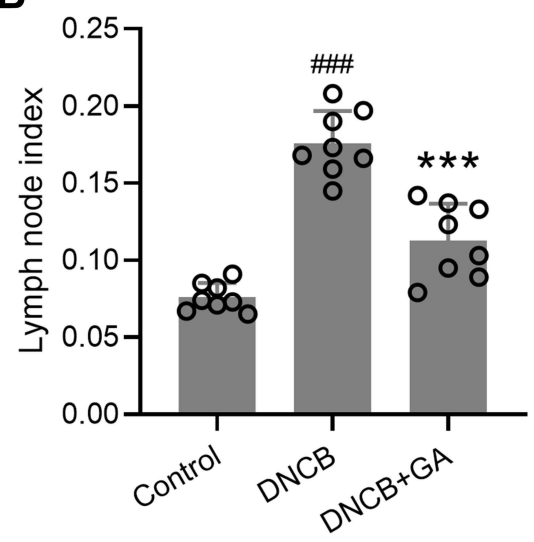

E

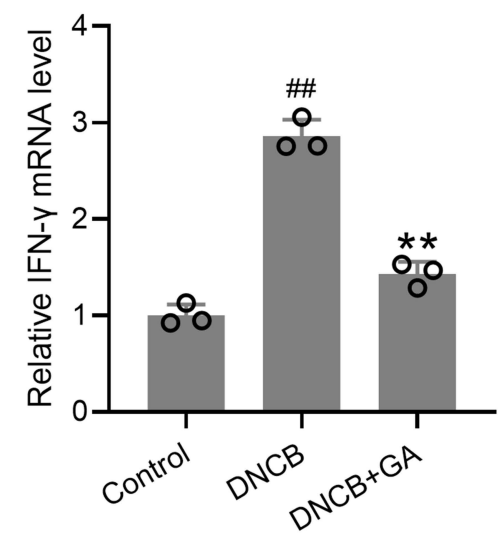

C

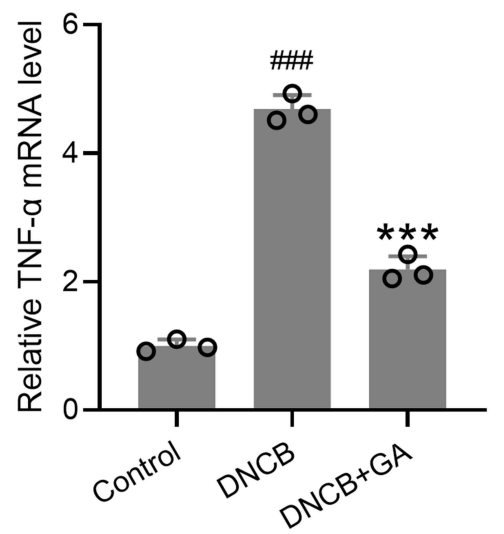

F

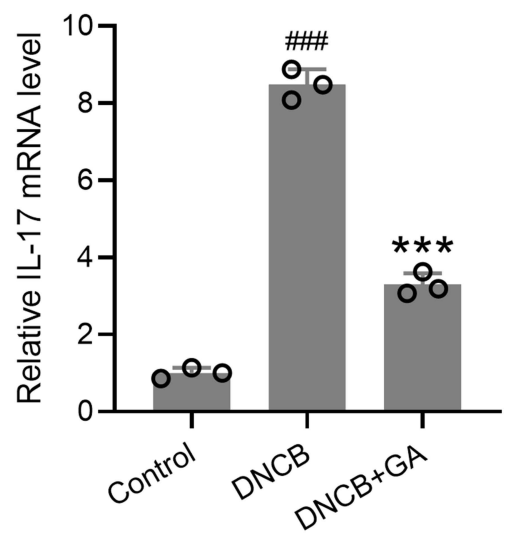

Figure 3 Effects of gallic acid on the weights of lymph node and the expression of various pathogenic factors in the lymph node. The lymph nodes were excised on day 43 and the weight were compared $(\mathbf{A})$ to calculate the lymph node index $(\mathbf{B})$. The total RNA was isolated and quantitative real time polymerase chain reaction was used to measure them RNA expressions of TNF- $\alpha(\mathbf{C})$, IL-4 (D), IFN- $\gamma(\mathbf{E})$, IL-I7 (F) in the lymph node tissues. Mean \pm SD was used to shown the data. \#P $<0.0$ I, \#\# $<0.00$ I compared to control, ${ }^{* *} \mathrm{p}<0.01$, ***p $<0.001$ compared to DNCB group. One-way ANOVA followed Dunn's multiple comparisons test, and Kruskal-Wallis test following by Mann-Whitney $U$-tests.

identify more molecular markers will help AD precision treatment, especially for diagnosis. Families with atopic diseases, including atopic dermatitis, asthma and allergic rhinitis, have family aggregation and may look for some breakthroughs from genetics. ${ }^{20}$ Nevertheless, therapy targeting inflammatory modulation is needed, so our study looked at whether GA could be effective on $\mathrm{AD}$ animal models in terms of inflammatory modulation.

Several mouse models have been used for $\mathrm{AD}$ research, like models induced by epicutaneous application of sensitizers, transgenic mice models, as well as mice that spontaneously develop AD-like skin inflammation including Nc/Nga mouse. ${ }^{21}$ The AD model in our study mimics the AD-like skin inflammation in terms of the thickness and inflammatory factors secreted. However, it could not in any way mimic the condition of $\mathrm{AD}$ in human; thus, further pre-clinical studies are still in need to prove the potential effect of GA on AD.
GA has been found to regulate various cellular pathways, such as the bone morphogenetic protein 2 (BMP2)-Smad1/5/8 signaling, ${ }^{22}$ c-Jun N-terminal kinases 2 (JNK2) signaling, ${ }^{23}$ TGF- $\beta /$ Smad 3 signaling, ${ }^{24}$ and histone deacetylase 1 or histone deacetylase $2 .{ }^{25}$ Its spectral anti-inflammatory function makes it clinically valuable. Our results showed that the four inflammatory factors in the lymph nodes were generally reduced under the action of GA, which was also consistent with its known function, and comparable to the positive control of Dexamethasone. Not only in the lymph nodes but also in situ, inflammatory factors significantly decreased upon GA treatment, and the rise of IL-10 and TGF- $\beta$ may be due to the action of Treg cells. GA may activate Treg to produce more inhibitory effects of active molecules, thus secreting more IL10 and TGF- $\beta$, which further supports the anti-inflammatory and immuno-modulatory effects of GA. Another study reported that unbalanced levels of IL-10 and TGF- $\beta$ in neutrophils contribute to an increase in inflammatory cytokine 
A

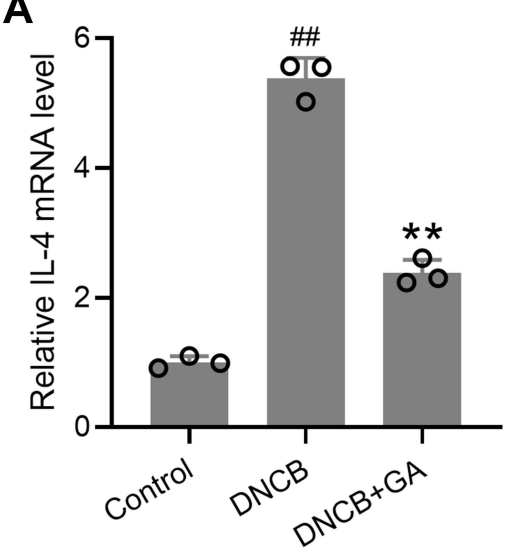

D

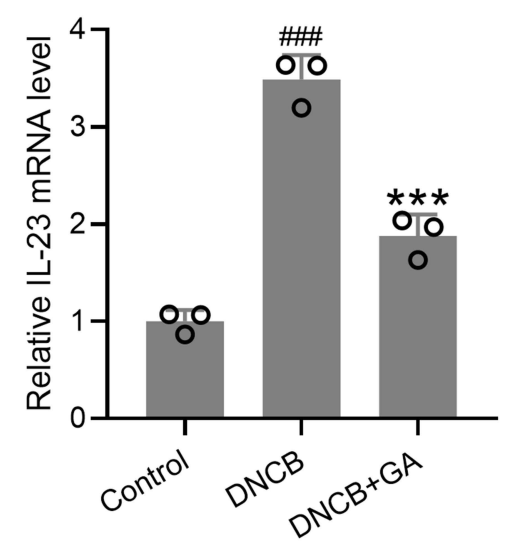

B

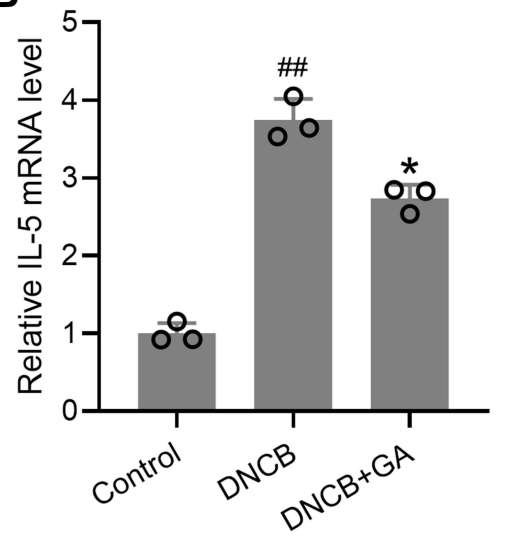

E

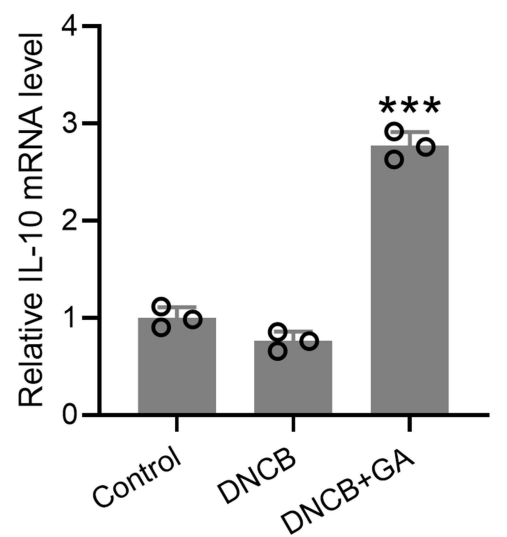

C

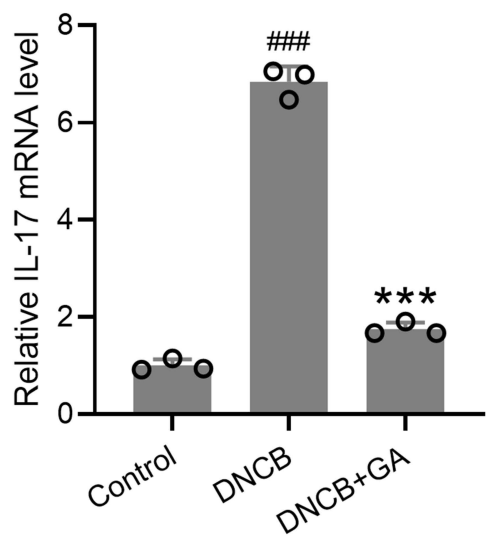

$\mathbf{F}$

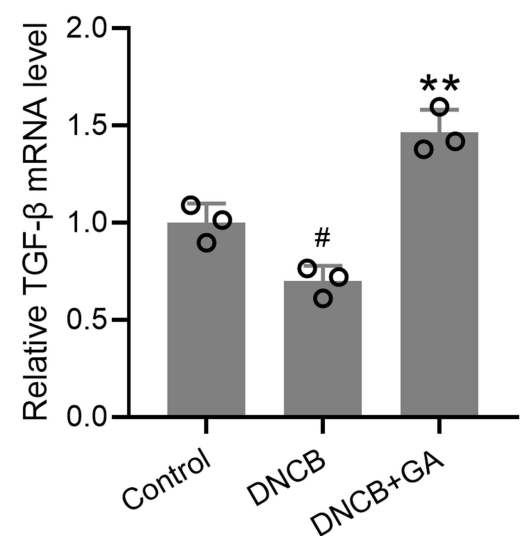

Figure 4 Effects of gallic acid on the expression of various pathogenic factors in the ear. The ears were excised on day 43 and quantitative real time polymerase chain reaction was used to measure the mRNA expressions of IL-4 (A), IL-5 (B), IL-I7 (C), IL-23 (D), IL-I0 (E) and TGF- $\beta$ (F) in the ear tissues. Mean \pm SD was used to shown the data. \#p $<0.05, \# p<0.01$, \#\# $<0.001$ compared to control, ${ }^{*} p<0.05$, ${ }^{*} p<0.01$, **** $<0.001$ compared to DNCB group. Kruskal-Wallis test following by MannWhitney U-tests.

expression in childhood obesity. ${ }^{26}$ It is well established that Treg cells can suppress immune responses via the production of IL-10 and TGF- $\beta .{ }^{27}$ Thus, GA could be a systemic candidate drug for $\mathrm{AD}$ through modulating IL-10 and TGF- $\beta$. In an allergic rhinitis mouse model, GA also showed an antiinflammation effect, which alleviated the nasal allergic symptoms with a decrease of IL-4, IL-13 and IL-17 in nasal lavage fluid. ${ }^{12}$ Thus, it is predictable that GA might also have an effect on $\mathrm{AD}$ immune system as previously reported. ${ }^{28}$ However, as $\mathrm{AD}$ is always sustained for a long time, the safety of GA for long-term use still needs investigation, and it also needs to be used with caution if there is a constant down-modulation of the immune system resulting from the GA treatment.

Th17 is an auxiliary T-cell differentiated by Th0 cells stimulated by IL-6 and IL-23, which mainly secretes IL-17, IL-22 and other inflammatory factors, and ROR is an important transcription factor for the fate decision of Th17. Th17 cells play an important role in the autoimmune system, they are a class of Th cell subgroups and are associated with many inflammatory responses including the occurrence and development of AD. Numerous studies have shown that IL-17 is associated with experimental autoimmune encephalitis (EAE), asthma, rheumatism, dermatitis, etc. Up-regulation of Th17 cells increases the risk of developing allergic rhinitis. Our experimental results also illustrate the involvement of Th17 in AD. Stimulated by TCR signals, regulated T cells can inhibit the activation and proliferation of a variety of cells, including effective T cells, B cells, natural killer cells (natural killer, NK) and dendritic cells. Our results showed that Th17 was activated in the DNCB-induced model and may not be able to regulate other $\mathrm{T}$ cell functions properly, while GA could inactivate Th17, enabled them function normally.

\section{Conclusion}

In the DNCB-induced AD-like mouse model, GA could alleviate the skin inflammation through immunomodulation of Th17. Therefore, GA might have the potential to treat $\mathrm{AD}$ in clinic. 
A

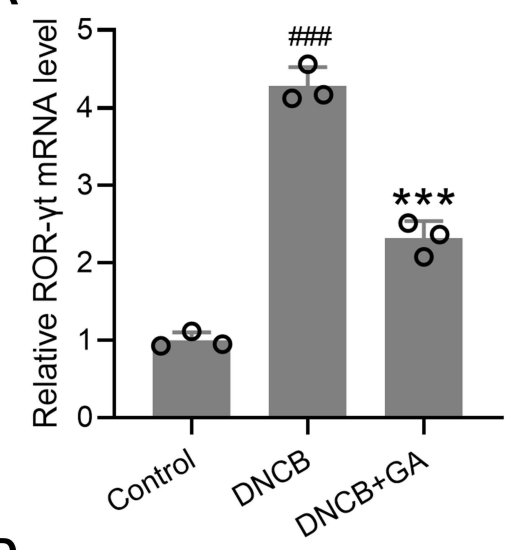

D

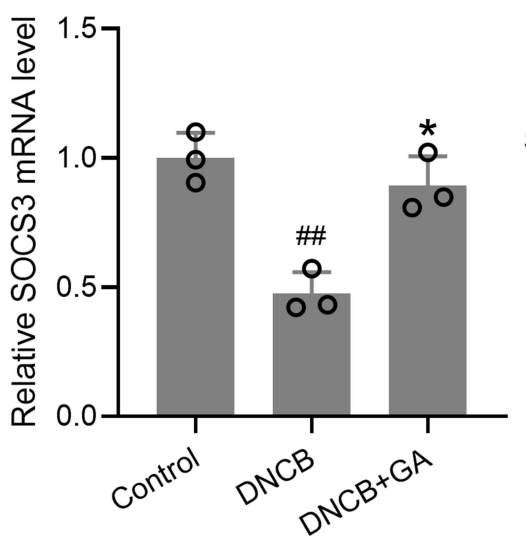

B

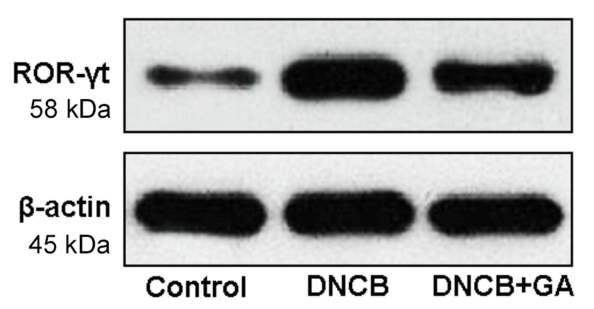

C

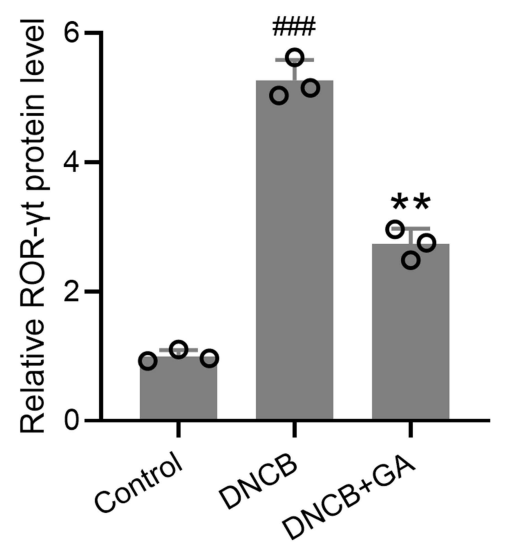

F

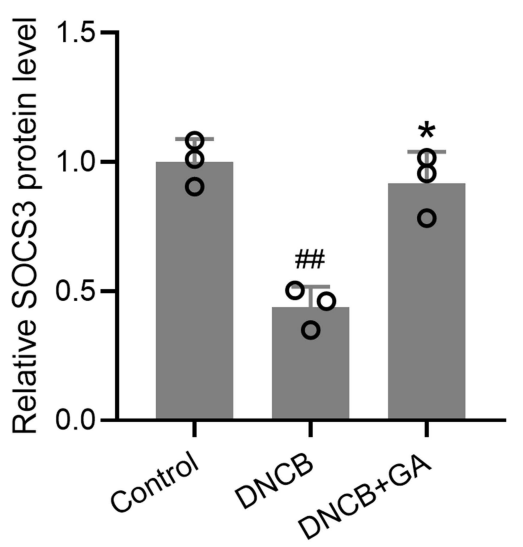

Figure 5 Effects of gallic acid on the ThI7 mediated immune responses in DNCB induced atopic dermatitis-like mice. The ears were excised on day 43 and quantitative real time polymerase chain reaction was used to measure the mRNA expressions of ROR- $\gamma \mathrm{t}(\mathbf{A})$ and SOCS3 (D) in the ear tissues. Western blotting was used to measure the protein levels of ROR- $\gamma \mathrm{t}(\mathbf{B})$ and SOCS3 (E) in the ear tissues. The expressions were normalized to control $(\mathbf{C}$ and $\mathbf{F})$. Mean \pm SD was used to shown the data. \#\# $<0.01$, \#\# $<0.001$ compared to control, ${ }^{*} \mathrm{p}<0.05$, ${ }^{*} \mathrm{p}<0.01$, ${ }^{* * *} \mathrm{p}<0.00 \mathrm{I}$ compared to DNCB group. Kruskal-Wallis test following by Mann-Whitney U-tests.

\section{Funding}

There is no funding to report.

\section{Disclosure}

The authors declare that they have no conflicts of interest.

\section{References}

1. Kim BE, Leung DYM. Significance of skin barrier dysfunction in atopic dermatitis. Allergy Asthma Immunol Res. 2018;10(3):207-215. doi:10.4168/aair.2018.10.3.207

2. Luger T, Amagai M, Dreno B, et al. Atopic dermatitis: role of the skin barrier, environment, microbiome, and therapeutic agents. J Dermatol Sci. 2021;102(3):142-157. doi:10.1016/j.jdermsci.2021.04.007

3. Gooderham MJ, Hong HC, Eshtiaghi P, Papp KA. Dupilumab: a review of its use in the treatment of atopic dermatitis. J Am Acad Dermatol. 2018;78(3 Suppl 1):S28-S36. doi:10.1016/j.jaad.2017.12.022

4. Rodrigues MA, Torres T. JAK/STAT inhibitors for the treatment of atopic dermatitis. J Dermatolog Treat. 2020;31(1):33-40. doi:10.1080/ 09546634.2019.1577549

5. Cotter DG, Schairer D, Eichenfield L. Emerging therapies for atopic dermatitis: JAK inhibitors. J Am Acad Dermatol. 2018;78(3 Suppl 1): S53-S62. doi:10.1016/j.jaad.2017.12.019
6. Williams HC. Clinical practice. Atopic dermatitis. $N$ Engl $J$ Med. 2005;352(22):2314-2324. doi:10.1056/NEJMcp042803

7. Tene K, Kalyan Kumar M, Basveshwar G, et al. Polyphenolicrich compounds from dillenia pentagyna (Roxb.) attenuates the doxorubicin-induced cardiotoxicity: a high-frequency ultrasonography assisted approach. Front Pharmacol. 2021;12:624706. doi:10.3389/fphar.2021.624706

8. Sliwinska S, Jeziorek M. The role of nutrition in Alzheimer's disease. Rocz Panstw Zakl Hig. 2021;72(1):29-39.

9. Bhat PB, Hegde S, Upadhya V, Hegde GR, Habbu PV, Mulgund GS. Evaluation of wound healing property of Caesalpinia mimosoides lam. J Ethnopharmacol. 2016;193:712-724. doi:10.1016/j.jep.2016.10.009

10. Chao J, Cheng HY, Chang ML, et al. Gallic acid ameliorated impaired lipid homeostasis in a mouse model of high-fat diet-and streptozotocin-induced NAFLD and diabetes through improvement of beta-oxidation and ketogenesis. Front Pharmacol. 2020;11:606759. doi:10.3389/fphar.2020.606759

11. Tsang MS, Jiao D, Chan BC, et al. Anti-inflammatory activities of pentaherbs formula, berberine, gallic acid and chlorogenic acid in atopic dermatitis-like skin inflammation. Molecules. 2016;21(4):519. doi:10.3390/molecules21040519

12. Fan Y, Piao CH, Hyeon E, et al. Gallic acid alleviates nasal inflammation via activation of Th1 and inhibition of Th2 and Th17 in a mouse model of allergic rhinitis. Int Immunopharmacol. 2019;70:512-519. doi:10.1016/j.intimp.2019.02.025 
13. Yoon CH, Chung SJ, Lee SW, Park YB, Lee SK, Park MC. Gallic acid, a natural polyphenolic acid, induces apoptosis and inhibits proinflammatory gene expressions in rheumatoid arthritis fibroblast-like synoviocytes. Joint Bone Spine. 2013;80(3):274-279. doi:10.1016/j.jbspin.2012.08.010

14. Choi EJ, Debnath T, Tang Y, Ryu YB, Moon SH, Kim EK. Topical application of Moringa oleifera leaf extract ameliorates experimentally induced atopic dermatitis by the regulation of Th1/Th2/Th17 balance. Biomed Pharmacother. 2016;84:870-877. doi:10.1016/j. biopha.2016.09.085

15. Bai XY, Liu P, Chai YW, et al. Artesunate attenuates 2, 4-dinitrochlorobenzene-induced atopic dermatitis by down-regulating Th17 cell responses in BALB/c mice. Eur J Pharmacol. 2020;874:173020. doi:10.1016/j.ejphar.2020.173020

16. Fu X, Hong C. Osthole attenuates mouse atopic dermatitis by inhibiting thymic stromal lymphopoietin production from keratinocytes. Exp Dermatol. 2019;28(5):561-567. doi:10.1111/exd.13910

17. Goindi S, Kumar G, Kumar N, Kaur A. Development of novel elastic vesicle-based topical formulation of cetirizine dihydrochloride for treatment of atopic dermatitis. AAPS PharmSciTech. 2013;14 (4):1284-1293. doi:10.1208/s12249-013-0017-3

18. Singh AK, Khare P, Obaid A, et al. SUMOylation of ROR-gammat inhibits IL-17 expression and inflammation via HDAC2. Nat Commun. 2018;9(1):4515. doi:10.1038/s41467-018-06924-5

19. Shi D, Yang J, Wang Q, et al. SOCS3 ablation enhances DC-derived Th17 immune response against Candida albicans by activating IL-6/ STAT3 in vitro. Life Sci. 2019;222:183-194. doi:10.1016/j. 1fs.2019.03.009

20. Tanaka N, Koido M, Suzuki A, et al. Eight novel susceptibility loci and putative causal variants in atopic dermatitis. J Allergy Clin Immunol. 2021. doi:10.1016/j.jaci.2021.04.019
21. Jin H, He R, Oyoshi M, Geha RS. Animal models of atopic dermatitis. J Invest Dermatol. 2009;129(1):31-40. doi:10.1038/ jid.2008.106

22. Kee HJ, Cho SN, Kim GR, et al. Gallic acid inhibits vascular calcification through the blockade of BMP2-Smad1/5/8 signaling pathway. Vascul Pharmacol. 2014;63(2):71-78. doi:10.1016/j. vph.2014.08.005

23. Ryu Y, Jin L, Kee HJ, et al. Gallic acid prevents isoproterenol-induced cardiac hypertrophy and fibrosis through regulation of JNK2 signaling and Smad3 binding activity. Sci Rep. 2016;6:34790. doi:10.1038/srep34790

24. Hussein RM, Anwar MM, Farghaly HS, Kandeil MA. Gallic acid and ferulic acid protect the liver from thioacetamide-induced fibrosis in rats via differential expression of miR-21, miR-30 and miR-200 and impact on TGF-beta1/Smad3 signaling. Chem Biol Interact. 2020;324:109098. doi:10.1016/j.cbi.2020.109098

25. Jang YG, Ko EB, Choi KC. Gallic acid, a phenolic acid, hinders the progression of prostate cancer by inhibition of histone deacetylase 1 and 2 expression. J Nutr Biochem. 2020;84:108444. doi:10.1016/j. jnutbio.2020.108444

26. Medeiros NI, Mattos RT, Menezes CA, et al. IL-10 and TGF-beta unbalanced levels in neutrophils contribute to increase inflammatory cytokine expression in childhood obesity. Eur J Nutr. 2018;57 (7):2421-2430. doi:10.1007/s00394-017-1515-y

27. Levings MK, Bacchetta R, Schulz U, Roncarolo MG. The role of IL-10 and TGF-beta in the differentiation and effector function of T regulatory cells. Int Arch Allergy Immunol. 2002;129(4):263-276. doi:10.1159/000067596

28. Dubin C, Del Duca E, Guttman-Yassky E. The IL-4, IL-13 and IL-31 pathways in atopic dermatitis. Expert Rev Clin Immunol. 2021;17 (8):835-852. doi:10.1080/1744666X.2021.1940962

\section{Publish your work in this journal}

Clinical, Cosmetic and Investigational Dermatology is an international, peer-reviewed, open access, online journal that focuses on the latest clinical and experimental research in all aspects of skin disease and cosmetic interventions. This journal is indexed on CAS.
The manuscript management system is completely online and includes a very quick and fair peer-review system, which is all easy to use. Visit http://www.dovepress.com/testimonials.php to read real quotes from published authors. 